\title{
Topisches Colchicin gegen aktinische Keratose und Skin Field Cancerization
}

Sowohl eine photodynamische Therapie (PDT) als auch die topische Behandlung mit Colchicin können aktinische Keratosen und eine „Skin field cancerization“ (SFC) verringern.

Das Prinzip der „field cancerization" wurde bereits 1953 für orale Plattenepithelkarzinome (SCC) beschrieben $[1,2]$. Demnach treten neben einem SCC Hautregionen auf, die bereits subklinische und multifokale Veränderungen aufweisen, die aus genetisch veränderten Zellen bestehen. Bei einer aktinischen Keratose (AK) beschreibt die „Skin field cancerization" (SFC) angrenzende Hautflächen neben einer AK, welche die Basis für eine klonale Expansion von genetisch veränderten Zellen darstellen. Dies kann ein Grund sein für den erneuten Ausbruch einer AK oder für die Entstehung von weißem Hautkrebs nach einer AK in der gleichen Region.

Sowohl topische Behandlungen als auch photodynamische Therapien (PDT) werden bei der AK eingesetzt (- Abb. 1). Dass eine topische Therapie mit Colchicin (COL) und eine PDT ungefähr gleich effektiv sind, zeigt jetzt eine klinische Studie [1] mit 36 Teilnehmern. In der Studie wurde auch deutlich, dass beide Behandlungen eine SFC reduzieren können.

\section{Beide Methoden sind effizient}

Die Probanden wiesen alle drei bis zehn Läsionen einer aktinischen Keratose an den Unterarmen auf und hatten in den vergangenen sechs Monaten keine Therapie aufgrund der Erkrankung erhalten. Jeder Patient wurde an einem Unterarm mit einer photodynamischen Therapie (Kürettage, Okklusion mit Methylaminolävulinat, MAL, 8-minütige PDT mit $630 \mathrm{~nm}$ Wellenlänge), am anderen Unterarm mit topischem COL $(0,5 \%$ zweimal täglich für zehn Tage) behandelt.
Die Effizienz beider Methoden war vergleichbar. Mit der COL-Behandlung gab es bei $45 \%$ der Patienten eine Verringerung der Anzahl aktinischer Keratosen, mit PDT waren es $40 \%$. Eine totale Clearance erreichten sechs Patienten (17\%) der COL-Gruppe und sieben Probanden im MAL-PDT-Arm (19\%). Die Autoren beobachteten eine partielle Clearance ( $>50 \%$ ) bei 16 Unterarmen (44\%), die mit COL behandelt worden waren und bei 24 Unterarmen (67\%), die eine PDT erhalten hatten $(p=0,07)$.

Zudem gab es in beiden Gruppen eine Verringerung der histologischen Atypien, die mittels KIN-Score (keratinocyte intraepithelial neoplasia) bestimmt wurden (COL: $20 \%$; MALPDT: $28 \%)$. Nach beiden Behandlungen gab es weniger epitheliale Atrophien $(p<0,01)$. Die HScore-Analyse für die KI76-Expression zeigte eine $15 \%$ ige Reduktion (COL) bzw. eine $9 \%$ ige Reduktion (MAL-PDT). Laut Autoren könnte u.a. die Ki67-Expression als Marker dienen, um die SFCAktivität zu bestimmen.

In beiden Gruppen gab es (meist leichte) Nebenwirkungen (COL: 27 Patienten, PDT: 24 Patienten). Am häufigsten waren Erytheme und eine Krustenbildung.

\section{Fazit der Studienautoren}

Bisher ist Colchicin auf dem europäischen Markt für die Behandlung einer aktinischen Keratose nicht verfügbar. Dennoch stellt der Wirkstoff eine kostengünstige Therapie dar, die effizient und sicher ist.

\section{Das Wichtigste in Kürze}

Wie effizient sind topisches Colchicin und eine photodynamische Therapie bei der Behandlung der AK?

Beide Strategien sind sicher und effizient. Außerdem lässt sich damit eine Skin Field Cancerization verringern. Einschränkungen (unter anderem): Monozentrische Studie, Zuweisung zu den Therapien war nicht verblindet.

\section{Literatur \\ 1. Miola AC, Ferreira ER, Lima TRR, Schmitt JV, Abbade LPF, Miot HA (2018) Effectiveness and safety of $0.5 \%$ colchicine cream vs. pho- todynamic therapy with methyl aminolae- vulinate in the treatment of actinic keratosis and skin field cancerization of the forearms a randomized controlled trial. Br J Dermatol 179(5):1081-1087 \\ 2. Slaughter DP, Southwick HW, Smejkal W (1953) Field cancerization in oral stratified squamous epithelium; clinical implications of multicentric origin. Cancer 6:963-968}

hautnah 2019 $\cdot 18: 91$

https://doi.org/10.1007/s12326-019-

0329-5

(c) Springer-Verlag GmbH Austria, ein Teil von Springer Nature 2019

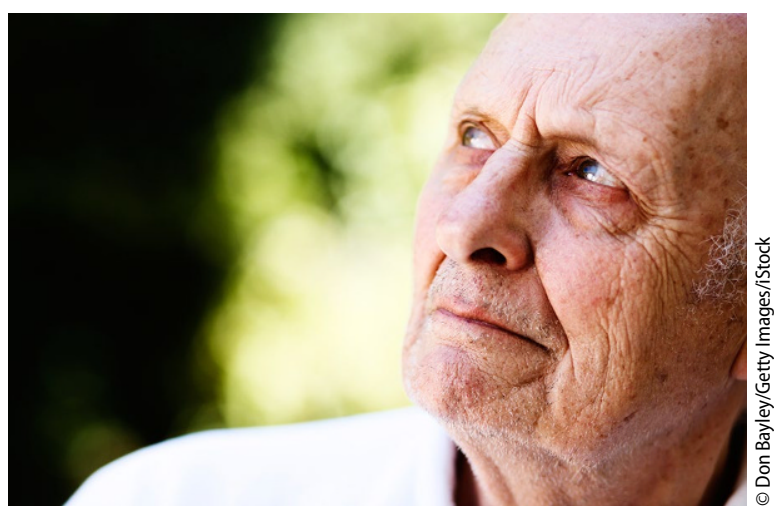

Abb. 1 \ Die aktinische Keratose kann photodynamisch oder topisch therapiert werden. (Symbolbild mit Fotomodell) 